尘理 学

\title{
刺激外周神经中枢端对鬼防御性 抬头反射的影响
}

范謹之 减谷民 胡三觉 王复周

过去目有人用光热刺激 家免悬子引起的抬头动作作 为研究药物傎痛作用的指 标 ${ }^{[1]}$. 我们把光热刺激改为 电刺激, 每当以电刺激家免 的悬中隔时, 家免便出现一 甚为典型的防护性的抬头动 作. 我们称之为抬头反射. 我们曾用这一反射的阈値作 为指标研究了针刺家免四肢 的某些 “穴位” 的镇痛作 用 ${ }^{[2]}$. 由于针刺 “穴位” 可 以被认为是一种外周刺激, 所以我们想用強度和作用时 间受控制的电流来刺激外周 神经的中枢端, 观察它们对

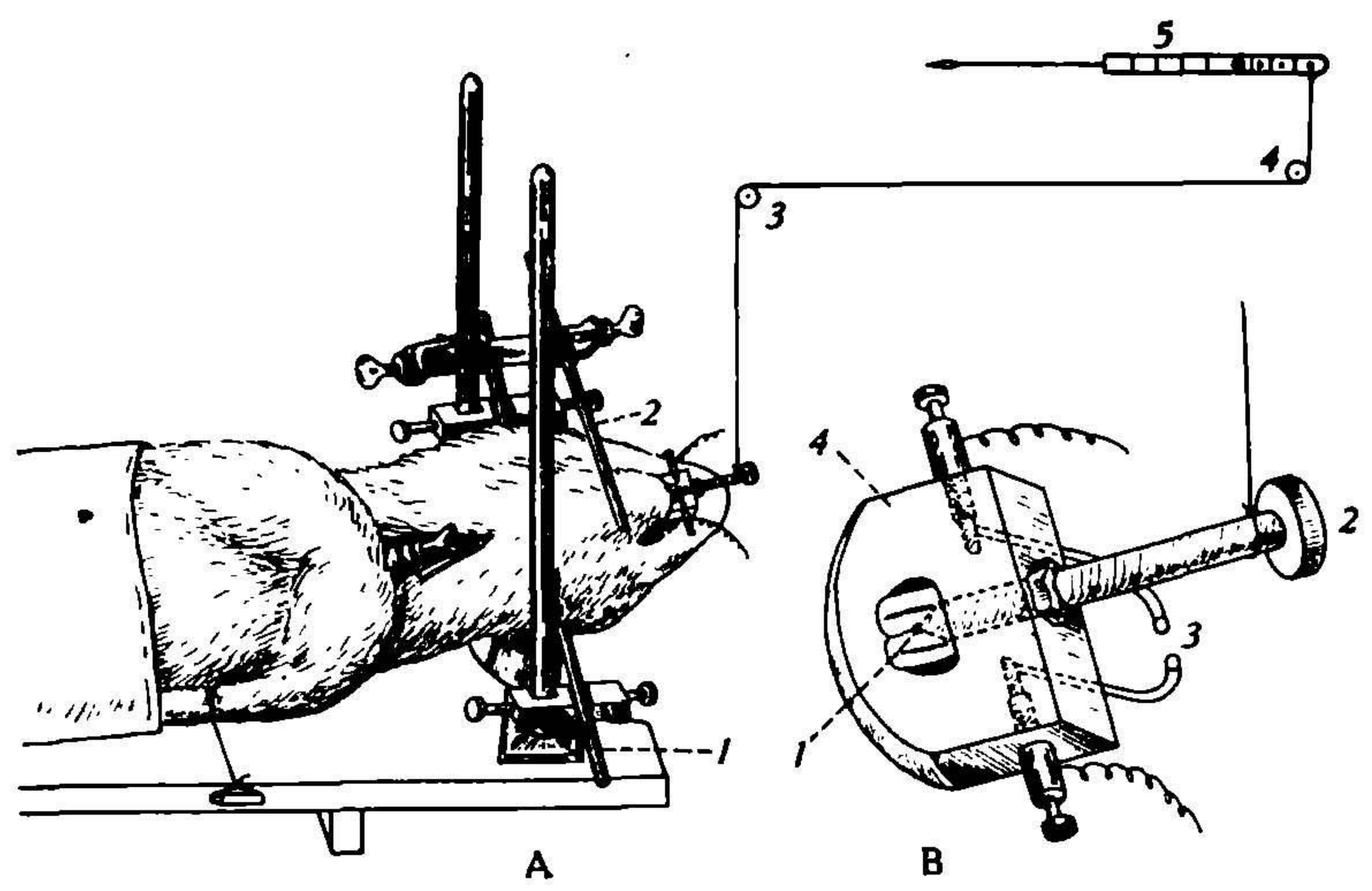

图 1 抬头反射记录方法示意图

A. 记录装目: 1. 固定免头用的尖头铜棒(顶在下頜关节陷宫中); 2. 限制低头

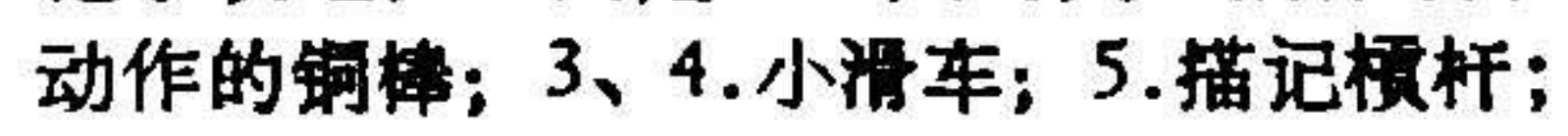

B. 特制基中隔刺激电极: 1. 在两上门茵的中央钻一个小凹䧄, 便于电极架的 固定; 2. 固定电极架的螺丝钉；3. 电极的游离端； 4 . 用塑料板制成的环形 电极架

免抬头反射的影响, 羊研究其影响机制, 借 以了解针刺“穴位”的镇痛机制。

家免以氮醴糖和氨基甲酸乙酯麻醉，头 用一对水平的铜棒固定起来, 但其抬头运动 却不受妨碍. 引起抬头反射的刺激电极装在 一环形的塑料板上, 将此环形塑料板套在上 门实上, 并用一螺丝钉固定紧。刺激电极的 自由端与冤罳中隔的两侧接触. 螺丝钉的一
端用线通过两个小滑车和杜杆相连, 借以把 抬头动作记录在记纹鼓上（图 1 ）。用方形 波刺激鼻中隔, 波宽为 1 毫秒左右, 強度为 1 -3 倍闻强度, 每次刺激的间隔时间为 1 秒, 每次刺激引起一个抬头反射.

对外周神经中枢端的刺激也用电子刺激 器. 刺激颈交感神经的频率为 20 次/秒, 刺 激其他神经为 50 次/秒。刺激的持续时间在 


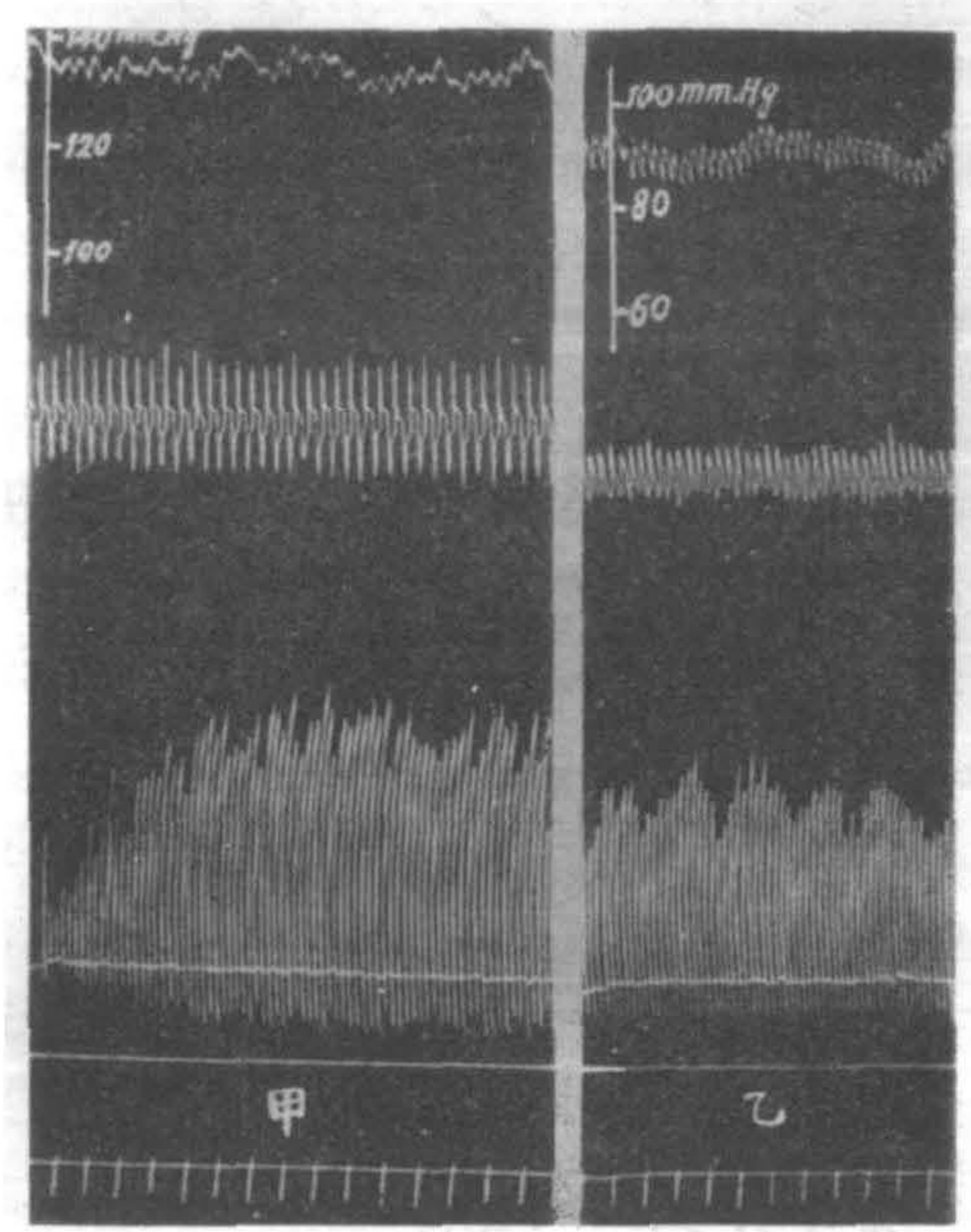

园 2 抬头反射的“类阶样现和”节律性波动

甲. “类阶栟现象”

免 45 号， $， 1.85$ 公斤。

曲线由上而下:

到总动瞅血压(水银拾压计记录法);

呼吸(气管攀管记录法);

抬头反射;

准备刺激外周神经的电磁标基线;

时标(每格 5 秒).

乙. 节律性波动

炭41㝍， $\sigma ， 1.92$ 公斤。

其他说明同甲

躯体神经为 5 秒, 在植物神经为 10 秒.

我们发现冤的抬头反射是一个甚为规律 性的反射，其国值约为 15 伏，在初期有 “类 阶梯现象”，然后其反射幅度便相当恆定，但 有节律性波动（图 2 ）。在实验连续进行 30 分钟以上时，未见娍弱，说明这一反射是不 易疲劳的. 它的感受野在悬中隔和悬唇沟， 因为当用 15 伏的电流剌激这些部位可引起 抬头反射时，若用 4 倍于此的強度刺激上、 下唇，口规和舌尖粘膜都无效. 用 3-5 伏 电流直接刺激眶下神经, 亦可引起抬头反

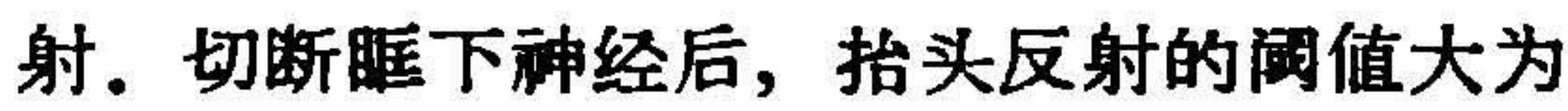

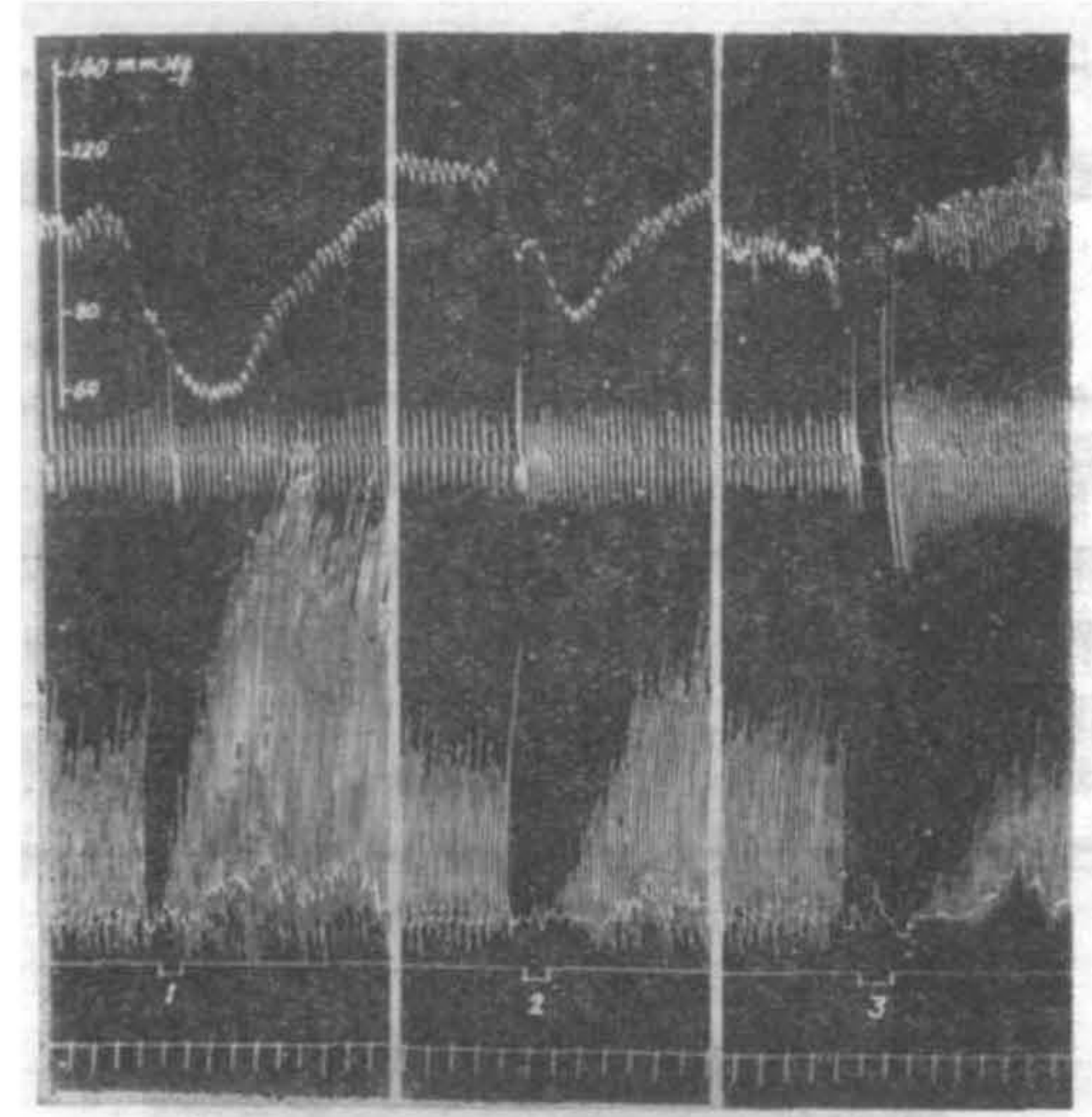

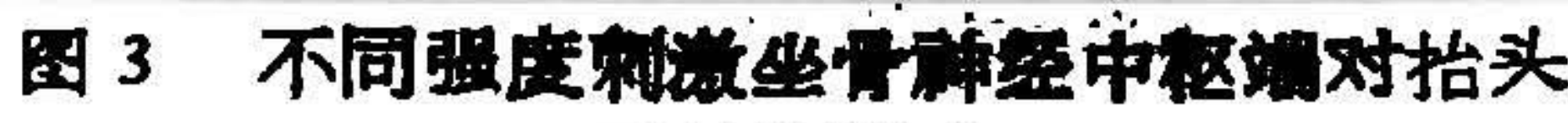
反射的影响

免 40 㝍, $\sigma^{\circ}, 1.75$ 公斤。

刺做记号 1，2、3 分別代衰用 20 伏、30 伏、 50 伏刺做坐骨动经中枢端 5 秒。

其他注解同国 2

升高, 由 15 伏升至 80 伏. 若再把嗅球切 除, 抬头反射便完全不能引起, 此时值接刺 激嗅神经亦可引起抬头反射. 以上都说明， 抬头反射的主要传入途径是眶下神经，在刺 激悬中隔的电流很強时, 也可通过咱神经完 成.

刺激坐骨神经的中枢端，显著地抑制抬 头反射. 随着刺激强度的增大，抬头反射可 完全被抑制（图 3 ）。坐骨神经的刺激停止 后，抬头反射又逐渐恢复，在开始恢复时多 出现“抑制后加强”现象 (图 3). 当坐骨神经 的刺激较强时，抬头反射的“抑制后加強”现 象不明显. 当把颈迷走神经切断后，剌激坐 骨神经中枢端对抬头反射的抑制作用便明显 減弱，表现为抑制的持续时间和抬头反射又 行恢复原有坘定高度所需的时间均缩短（表 1 及图 4). 切断颈交威神经和減压神经并 不影响刺激坐骨神经中枢端对抬头反射的抑 制作用，可见颈迷走神经在抑制抬头反射中 
表 1 经迷走神经切断前后，剌放坐骨神

经中枢端对抬头反舫抑制作用的比较

\begin{tabular}{|c|c|c|c|c|}
\hline & \multicolumn{2}{|c|}{$\begin{array}{l}\text { 拍头反射完全 } \\
\text { 抑制时间(称) }\end{array}$} & \multicolumn{2}{|c|}{ 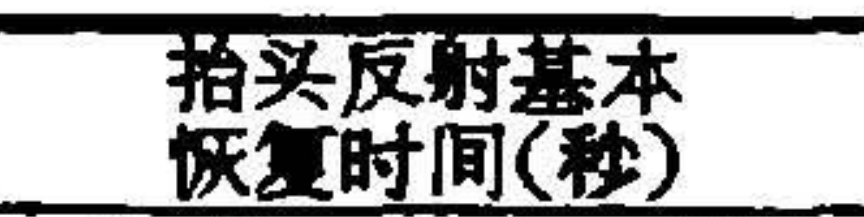 } \\
\hline & 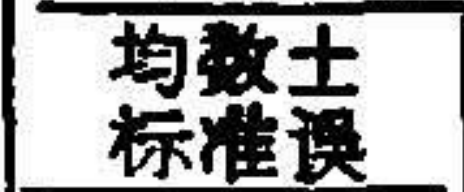 & $\begin{array}{l}\text { t测检 } \\
P \text { 蹎 }\end{array}$ & 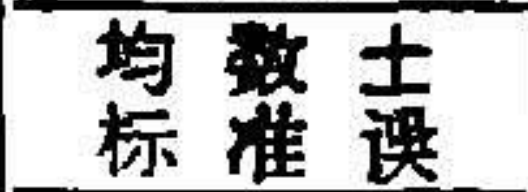 & $\begin{array}{l}t \\
P \text { 测器 } \\
\text { 值 }\end{array}$ \\
\hline 切断会迷走 & $8.29 \pm 0.76$ & & $43.72 \pm 7.24$ & \\
\hline 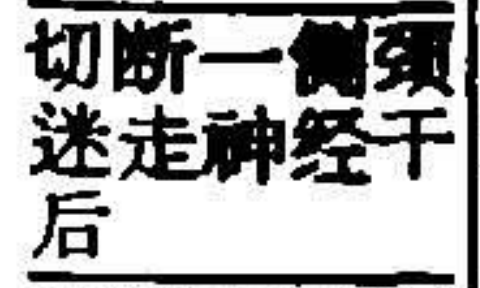 & $5.71 \pm 0.47$ & $P<0.05$ & $37.50 \pm 6.67$ & $P>0.05$ \\
\hline $\begin{array}{l}\text { 切断两 } \\
\text { 迷走象经行 } \\
\text { 后 }\end{array}$ & $3.79 \pm 0.82$ & $P<0.02$ & $26.21 \pm 6.67$ & $P<0.05$ \\
\hline
\end{tabular}

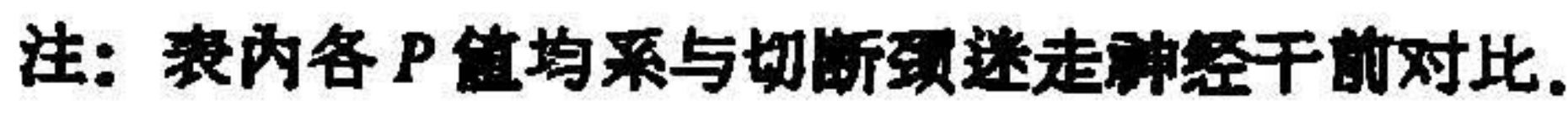

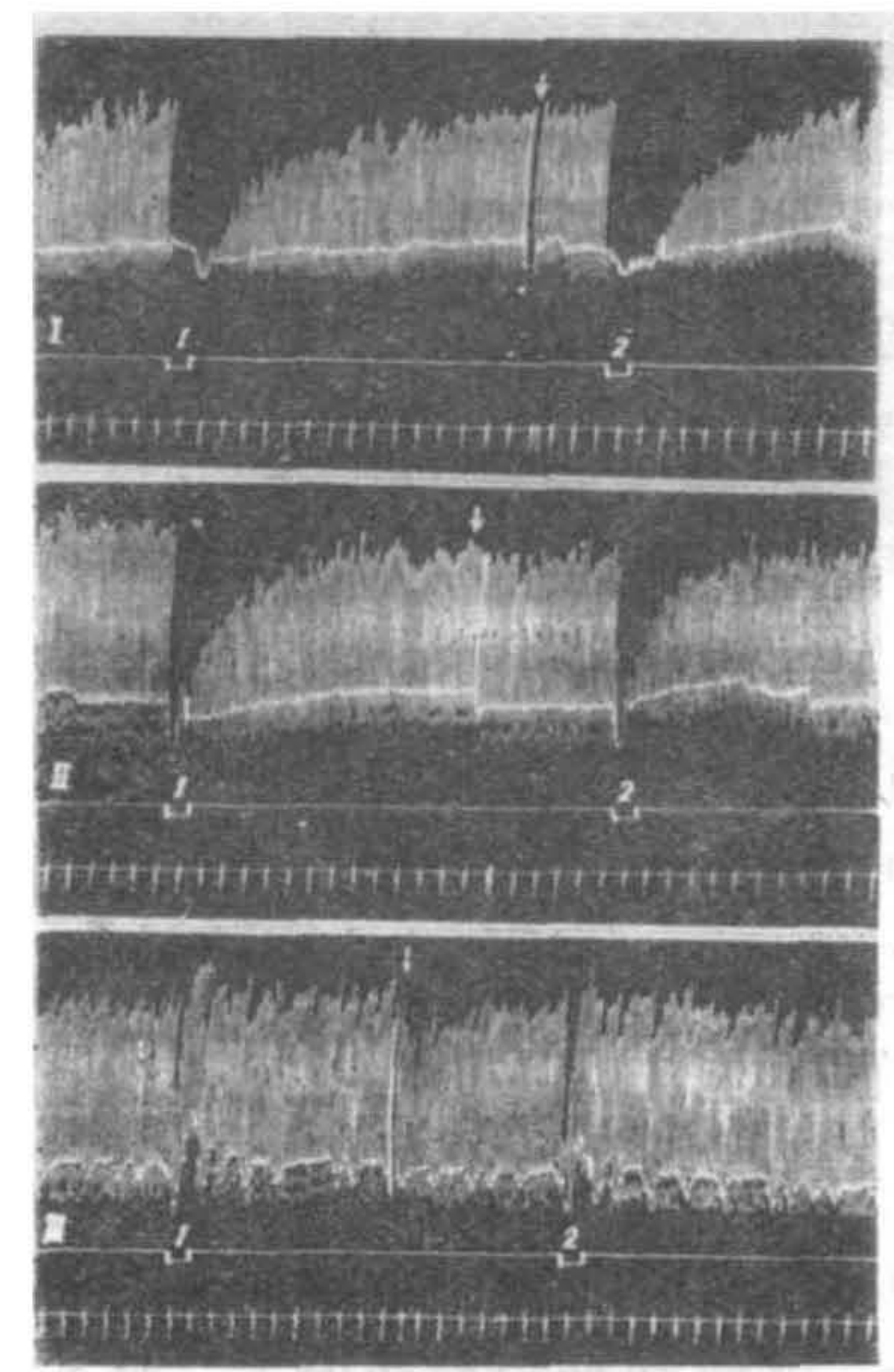

图 4 切断颈迷走神经干后，刋湤坐骨神 经中枢端对抬头反射的影响

色 30 号,, 2 公厅.

I一颈迷走神经干切断前;

II一切断一侧顽迷走神经干后;

III一切断㑂侧到迷走神经干后。

I、II、III 中的曲线由上而下: 抬头反射（有 处 表示停止刺激梨中隔 1 分钟); 刺激记号 1、2 均为刺激坐骨神经中枢端 5 秒, 強度 8 伏; 时 标(每恪 5 秒)

起一定作用。迷走神经的这一作用可为下 边实验进一步证实, 郎当值接刺激（一般用 5-10 伏的电压郎可）颈迷走神经的中枢端 时，抬头反射也受到明显的抑制（图 5)。根

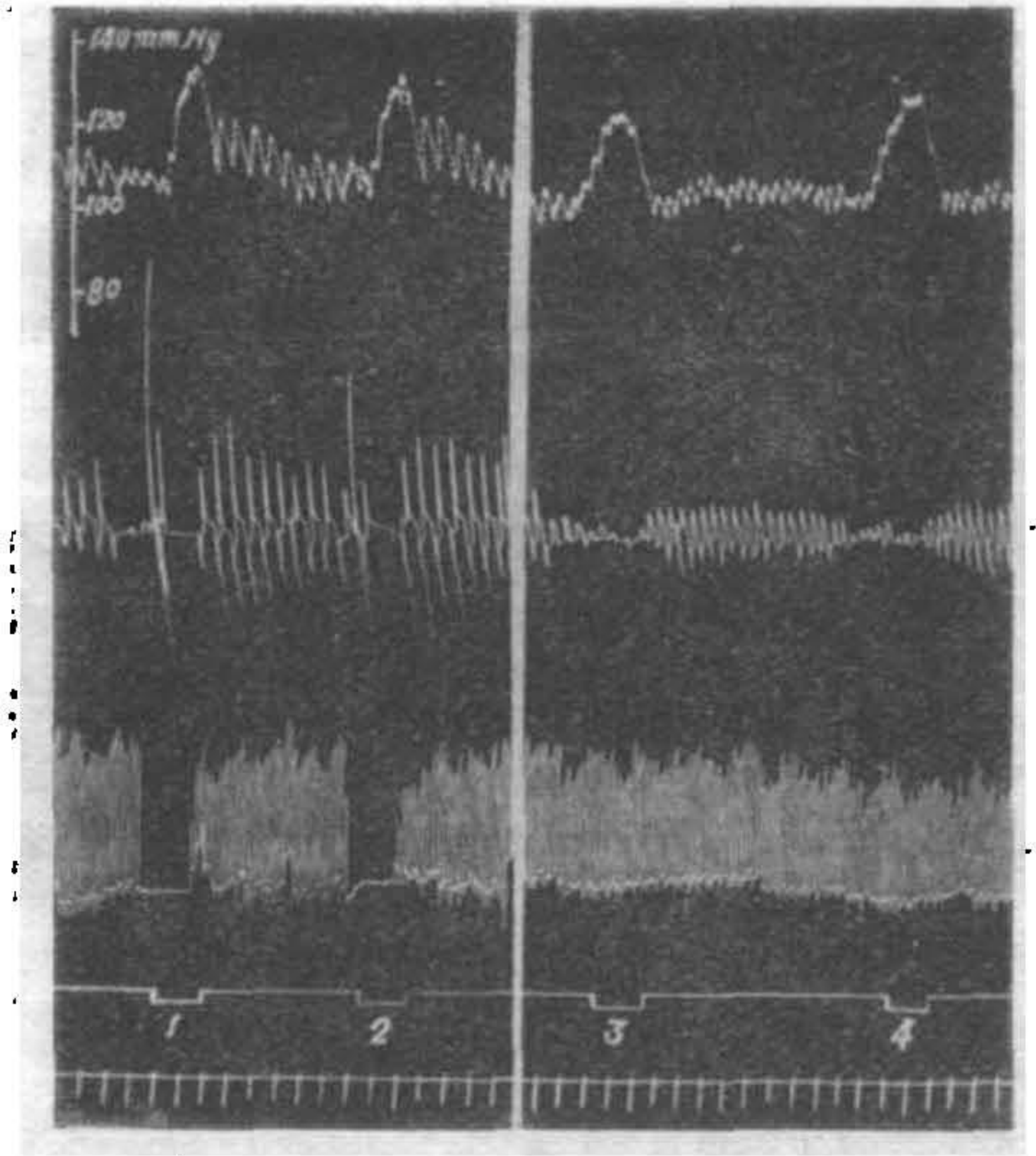

图 5 刺激到迷走神经中枢端与央臸总动 脉对抬头反舫影响的比较

免 41 号, Or, 1.92 公斤。

剌激记号 $1 、 2$ 均为刺制顽迷走神经中枢端 10 秒, 強度 10 伏; 刺激记号 3、4 均为来住颈总动脉阻 断血流 10 秒。

其他注解同国 2

据这些现象，我们推想刺激坐骨神经中枢端 可能会引起颈迷走神经的传入冲动增加, 这 些传入冲动加強了坐骨神经的传入冲动对抬 头反射的抑制作用。实验也证明如此，因为 当以电流刺激坐骨神经中枢端时，颈迷走神 经的传入冲动确实明显增加（图 6).

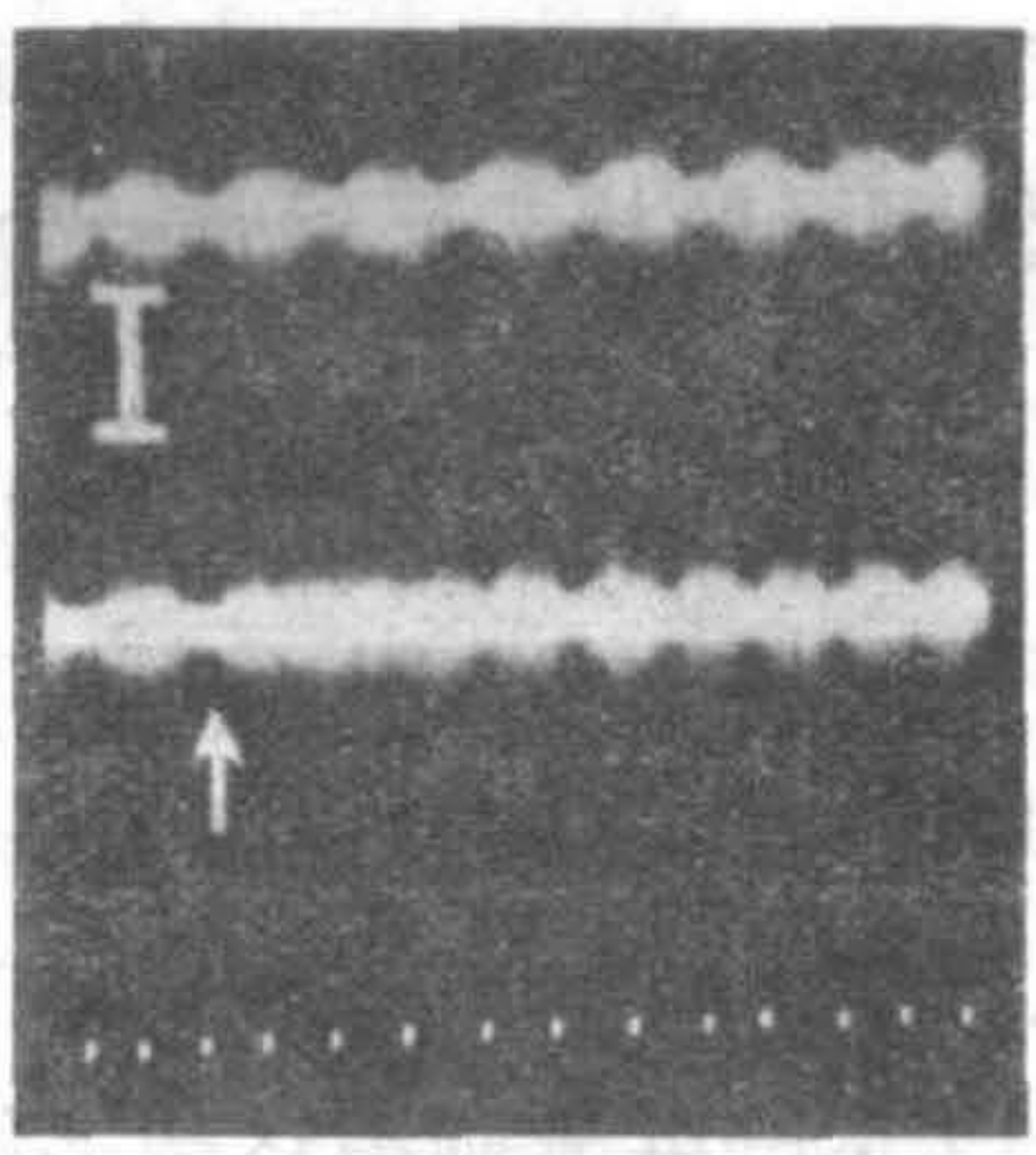

图 6 刺激坐骨神经中枢端对颈迷走神 经干传入冲动的影响

免 84 号, 万7, 2 公斤。 曲线由上而下: 预迷走神经干的传入冲动， I 代 表 25 微伏: 上段曲线的继续，有个处为开始刺 激坐骨神经 5 秒, 強度 5 伏; 时标(每格 1 秒) 
在有些实验中，我们还记录了动物的血 医变化，我们不能发现抬头反射的抑制与血 压变化之间有任何关系(图 2、3、5).

为了查明刺激坐骨神经中枢端时颈迷走 神经传入冲动龧加的原因，我们又做了下列 实验. 用注射器通过气管套管向肺內迅速打 入 30-40 毫升空气; 使肺膨胀，发现迷走 神经的传入冲动明显增加, 类似于刺激坐骨 神经中枢端时迷走神经传入冲动的变化. 通 过事先置于胃內的气球膨胀胃，则未见迷走 神经传入冲动㘿加. 另一方面, 当迷走神经 完整存在时，膨胀肺也可抑制抬头反射，膨 胀胃则对抬头反射无影响。根据上迅实验结 果, 我们认为迷走神经参与刺激坐骨神经中 枢端对抬头反射的抑制作用的机制可归纳如
下: 剌激坐骨神经中枢端引起了动物呼吸运 动的加强, 肺的晕张威受器受到剌激, 迷走 神经的传入冲动便增多，因之对抬头反射发 生了抑制作用。

刺激其他一些神经, 如胫神经、腓神 经、胎神经和內脏大神经的中枢端都对抬头 反射有不同程度的抑制作用, 或者其抑制作 用较弱，或者所需刺激电流较強。刺激诚压 神经和颈交感神经的中枢端和外周端对抬头 反射均无影响.

\section{考文缺}

[1] 金国章、胥柪，生理学报，21 [2]，150-157(1957).

［2]范谨之、胡三赏、王复周，全国中医经络针炎学术 座谈公负料选编，人民卫生出版社， 257-260， 1959 . 